\title{
Bifurcating combustion behaviour
}

\author{
R.O. Weber* \\ G.N. Mercer* \\ H.S. Sidhu*
}

(Received 7 August 2000)

\begin{abstract}
Many different combustion situations exhibit bifurcations. Some of these are merely artefacts of the models constructed, but others are of physical significance. This paper will discuss the calculation of this behaviour as it occurs in several situations; namely, spontaneous thermal ignition in a sphere, propagating flames and microgravity flame balls.
\end{abstract}

${ }^{*}$ School of Mathematics and Statistics, University College of UNSW, ADFA Canberra 2600, Australia. mailto:r.weber@adfa.edu.au, mailto:g.mercer@adfa.edu.au and mailto:h.sidhu@adfa.edu.au

${ }^{0}$ See http://anziamj . austms . org.au/V42/CTAC99/Webe for this article and ancillary services, (C) Austral. Mathematical Soc. 2000. Published 27 Nov 2000. 


\section{Contents}

1 Introduction $\quad$ C1466

2 Thermal Ignition in the Sphere $\quad$ C1467

3 Combustion Waves for Gases $(\mathrm{Le}=1$ ) and Solids (Le $\rightarrow$ () $\quad$ C1471

3.1 Governing Equations . . . . . . . . . . . . . . . C1471

3.2 Numerical Solutions. . . . . . . . . . . . . . . C1474

4 Flame Balls $\quad$ C1476

5 Conclusion $\quad$ C1478

References

$\mathrm{C} 1478$

\section{Introduction}

Mathematical models of combustion usually involve nonlinear source terms in the equations for energy and mass conservation. This is aside from the fluid dynamical nonlinearities which can be relevant in cases where an unknown flow is coupled to the combustion and must be solved for simultaneously. The non-linearities in the energy and mass equations tend to preclude ex- 
act analytical solutions for combustion problems, although it is sometimes possible to construct asymptotic solutions for certain parameter ranges.

On the other hand, the same non-linearities are responsible for quite interesting equilibrium response curves and also some remarkable dynamic behaviour. Phenomena such as extinction, ignition and pulsation can all be found in these mathematical models of combustion and seem to correspond reasonably well to behaviour observed with real chemical combustion reactions. One of the main ways of identifying this behaviour in the combustion models is to seek bifurcations in equilibrium curves and in other response diagrams. This paper will consider three combustion examples where this has been done numerically: spontaneous ignition in the sphere, traveling solid combustion waves, and microgravity flame balls. In each case it was not possible to obtain the bifurcation diagrams directly from the partial differential equations. Rather, it was necessary to first simplify the problem and conduct some preparatory analysis. Only then, by the use of path following methods, was it possible obtain the bifurcation diagrams and unravel the interesting non-linear behaviour characteristic of mathematical models of combustion.

\section{Thermal Ignition in the Sphere}

If we seek a balance between the effect of diffusional fluxes of temperature and an exothermic reaction $f(u)$ in a homogeneous sphere, then it can be shown (Frank-Kamenetskii [5], Zeldovich [13, pp.158-161, e.g.], Weber, Wake 
and Balakrishnan [10] and references therein) that the non-dimensional temperature variable $u$ must satisfy

$$
\frac{d^{2} u}{d r^{2}}+\frac{2}{r} \frac{d u}{d r}+\lambda e^{-1 / u}=0
$$

This equation represents a steady-state and non-dimensionalised statement of the principle of conservation of energy and as such is the starting point for many investigations into thermal ignition theory. Naturally this equation will need to be solved for the temperature $u$, as a function of the radial coordinate $r$ and the parameter $\lambda$, subject to the appropriate Dirichlet boundary conditions.

In order to keep the subsequent analysis and presentation of results reasonably simple, we shall restrict our study to the case where the exterior is held at a constant temperature; called the ambient temperature and denoted $u_{a}$. Due to the use of dimensionless parameters, we can assume, without loss of generality, that the sphere has unit radius. Hence our boundary conditions can be written as

$$
\begin{aligned}
\left.\frac{d u}{d r}\right|_{r=0} & =0, \\
u(1) & =u_{a} .
\end{aligned}
$$

The first of these is merely a statement requiring bounded solutions at the centre of our spherically symmetric domain. Additionally, it should be noted 
that our choice of reasonably simple boundary conditions is not essential for the surprisingly complex solutions which we will display. It does, however, make our task of presenting, explaining and analysing the results somewhat easier and (hopefully) less confusing.

Solutions of equation (1) subject to boundary conditions (2) need to be constructed numerically (with the possible exception of a series approach for the simple exponential reaction as detailed in Frank-Kamenetskii [5]). Certainly there has been considerable effort in this direction and a consistent picture of the results has emerged. For sufficiently large $\lambda$ and for some $0<u_{a}<\frac{1}{4}$, there is found to exist one low temperature solution, stable to temporal perturbations, and one high temperature solution, also stable to temporal perturbations. In addition, there exists a large number of intermediate solutions, all of which are unstable to temporal perturbations. This is best displayed and understood with a bifurcation diagram, plotting the maximal steady state temperature $u(0)$ as a function of a bifurcation parameter; for example $u_{a}$, and holding the other parameter, $\lambda$, constant. In Figure 1 (after Weber et al. [12]), the result for $\lambda=10^{12}$, a realistic value for milk powder [8], is shown and it can be clearly seen that for certain values of $u_{a}$, there can exist upto seven intermediate steady states; all of which turn out to be unstable if analysed by a linear, temporal, stability analysis or if analysed numerically. This bifurcation diagram was obtained using a path following routine implemented in MATLAB ${ }^{\mathrm{TM}}$ and verified with a routine from AUTO97 [4]. 


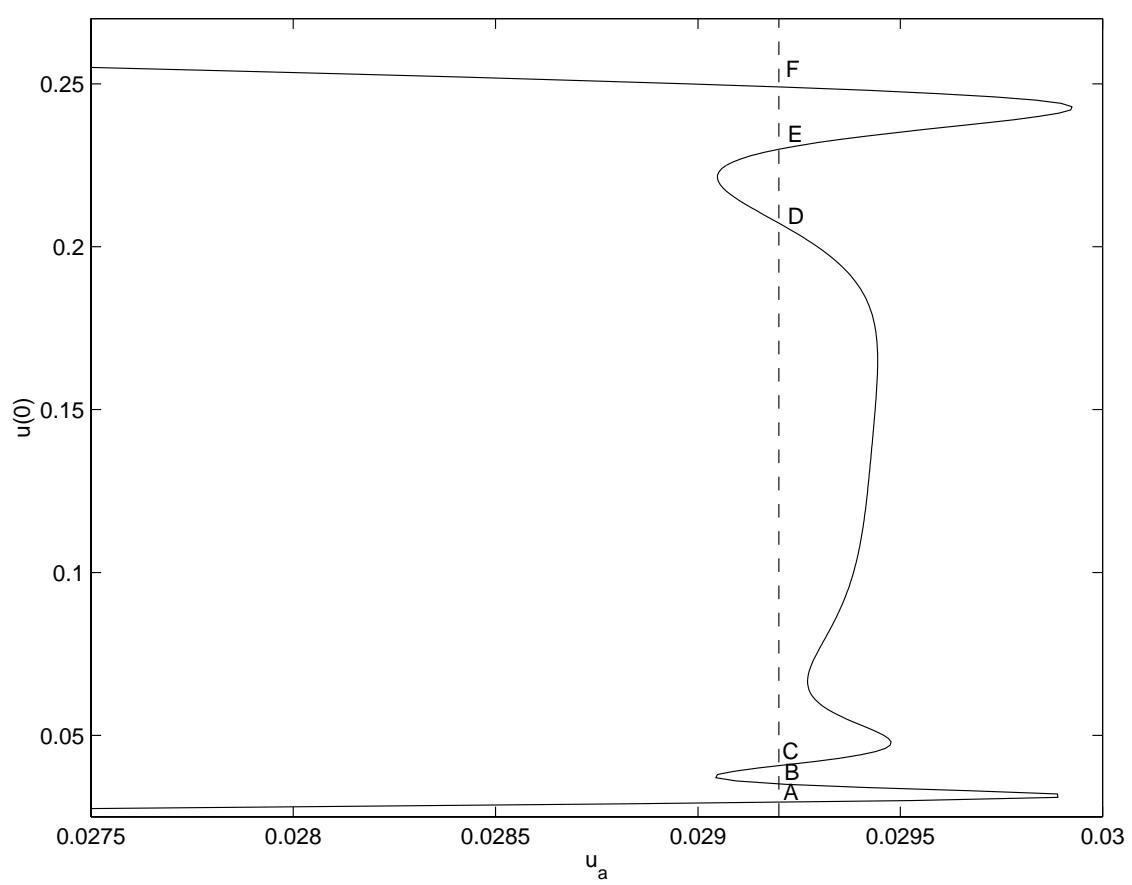

FIGURE 1: Bifurcation diagram showing the central temperature $u(0)$ as a function of ambient temperature $u_{a}$ for fixed $\lambda=10^{12}$ for a unit sphere. For the particular value $u_{a}=0.0292$, shown by the dotted line, five unstable, intermediate, steady state profiles exist and are labelled B, C, D, E and F. The profile labelled $\mathrm{A}$ is the lowest and is stable. The uppermost profile is also stable but cannot be accommodated within the scaling shown here. 
3 Combustion Waves for Gases $(\operatorname{Le}=1)$ and Solids $($ Le $\rightarrow \infty)$

\section{Combustion Waves for Gases $(\mathrm{Le}=1)$ and Solids $($ Le $\rightarrow \infty)$}

Also of considerable interest are possible bifurcations in the wave speed of combustion waves, as first observed by Shkadinskii et al. [9], analysed by Matkowsky and Sivashinsky [7] and subsequently followed up by others, including Bayliss and Matkowsky [1]. The original analysis was carried out for a delta-function model of the reaction and all the subsequent work has utilised the traditional choice for non-dimensional temperature, $\theta$. While all the research has given consistent answers, there is still a small element of doubt concerning the appearance of chaotic combustion waves in the original partial differential equation system. Independent confirmation of these results, particularly when these are obtained with a significantly different nondimensional temperature and a completely different numerical method (e.g. Weber et al. [11]), provides useful evidence that the results are not merely an artefact of the delta-function model (analysis) or a particular method for discretising the equations (numerical).

\subsection{Governing Equations}

Consider a premixed fuel arranged in a one-dimensional configuration with possible, bulk heat loss. An example would be a heavily insulated, long cylinder of a pyrotechnic mixture. Assuming that the rate of exothermic 


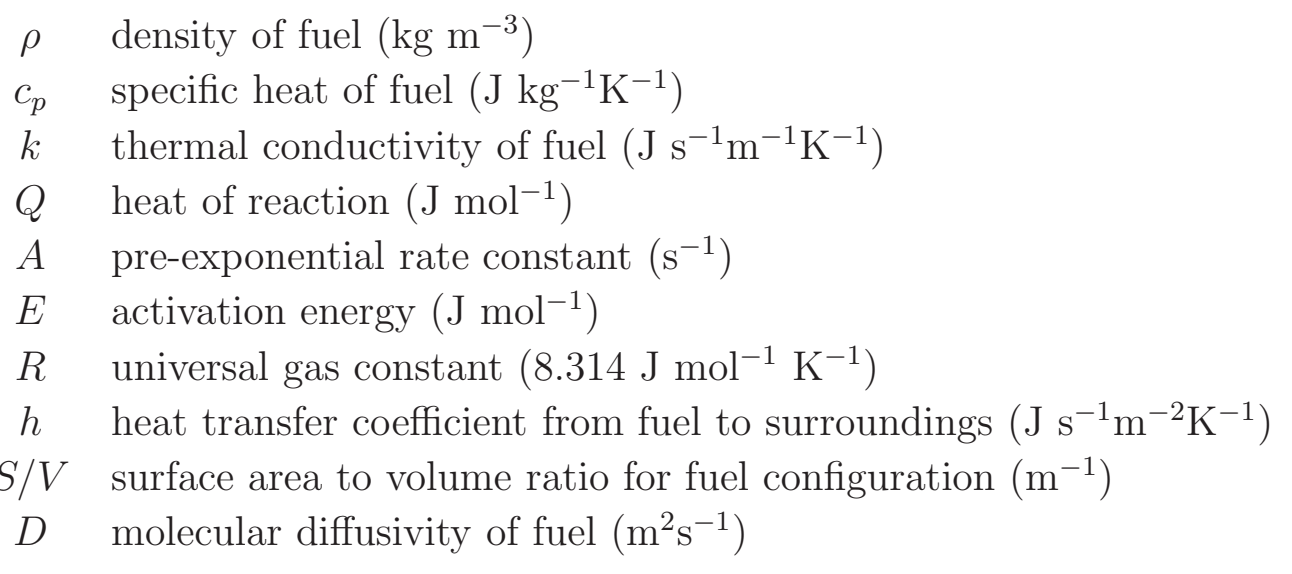

combustion is well described by the Arrhenius Law, it follows from the conservation of energy and chemical species that governing equations are

$$
\begin{aligned}
\rho c_{p} \frac{\partial T}{\partial t} & =k \frac{\partial^{2} T}{\partial x^{2}}+Q A Y e^{-E / R T}-\frac{h S}{V}\left(T-T_{a}\right) \\
\frac{\partial Y}{\partial t} & =D \frac{\partial^{2} Y}{\partial x^{2}}-A Y e^{-E / R T}
\end{aligned}
$$

In these equations, the temperature (in Kelvin) and concentration (in mol $\mathrm{m}^{-3}$ ) of the fuel are denoted by $T$ and $Y$ respectively; and $x$ and $t$ describe space and time coordinates. All the other quantities are constants (though some can be easily controlled in any given experiment). 
Defining the non-dimensional temperature to be

$$
u=\frac{R T}{E},
$$

and rescaling the space and time coordinates where $x$ has had $\left(Q A Y_{0} R / k E\right)^{\frac{1}{2}}$ and $t$ has had $\left(Q A Y_{0} R / \rho c_{p} E\right)$ absorbed respectively, the two coupled equations can be written in non-dimensional form as

$$
\begin{aligned}
& \frac{\partial u}{\partial t}=\frac{\partial^{2} u}{\partial x^{2}}+y e^{-1 / u}-\ell\left(u-u_{a}\right) \\
& \frac{\partial y}{\partial t}=\frac{1}{\operatorname{Le}} \frac{\partial^{2} y}{\partial x^{2}}-\beta y e^{-1 / u}
\end{aligned}
$$

The new parameters are

$$
\begin{aligned}
u_{a} & =R T_{a} / E, \\
\ell & =h S E / V Q A Y_{0} R, \\
\mathrm{Le} & =k / D \rho c_{p}, \\
\beta & =\rho c_{p} E / R Q Y_{0} .
\end{aligned}
$$

Of these parameters, $u_{a}$ and $\ell$ are obvious control variables for laboratory experiments as they are the non-dimensional equivalents of the ambient temperature $\left(T_{a}\right)$ and the heat coefficient $(h)$ respectively. These can both be confidently manipulated in the laboratory. $Y_{0}$ is a reference concentration. The Lewis number, Le, has two particularly significant values; Le around one 
for gaseous fuels and Le $\rightarrow \infty$ for solid fuels, such as pyrotechnics. The last parameter, $\beta$, can be significantly larger than unity for selected fuels and this gives the circumstances leading to a bifurcation in the combustion wave speed.

\subsection{Numerical Solutions}

Aside from being able to find asymptotic solutions; e.g. Weber et al. [11], we can use a numerical approach to determine the speed of any combustion waves for $\mathrm{Le}=1$ and $\mathrm{Le} \rightarrow \infty$ as a function of $\beta$.

The numerical results, shown in Figure 2 for Le $\rightarrow \infty$ and with zero heat loss, indicate that there ceases to be a permanent form travelling wave with a single wave speed when $\beta$ becomes larger than (approximately) 6.5 . This observation accords with that of Matkowsky and Sivashinsky [7] and subsequently Bayliss and Matkowsky [1], although there are significant differences in the precise details of the models and in the numerical methods. Consideration of the stability of the traveling combustion wave fronts suggests a period doubling route to chaos for the premixed solid fuel as $\beta$ increases beyond 6.5 ; i.e., as the exothermicity of the reaction is decreased. 


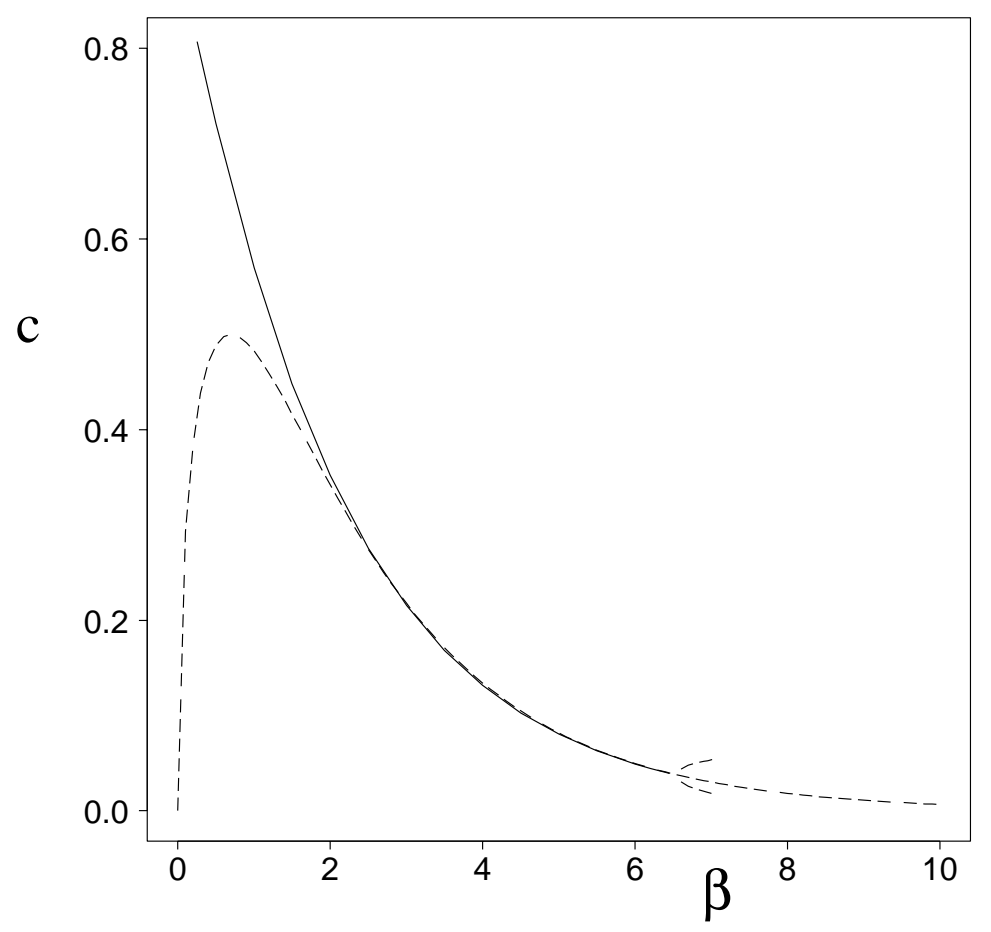

Figure 2: Computed wave speed for Le $\rightarrow \infty$, showing bifurcation at $\beta$ approximately 6.5 which reflects an oscillating wave speed. The solid line is the numerical result and the dashed line is an asymptotic approximation. After Weber et al. [11]. 


\section{Flame Balls}

Recent 'drop-tower' and 'space shuttle' experiments have conclusively demonstrated that spherical flame structures can persist for long times; at least nine minutes, [6]. It so happens that a spherical flame model was examined many years ago by Zeldovich [13, pp.327-331, e.g.] in the hope of constructing stable stationary solutions. While a stationary solution was found, it turned out to be unstable to perturbations. It wasn't until the 1990's that Buckmaster et al established that heat losses of any kind can act as a stabilising mechanism [2]. Experiments in 1996 and 1997 have shown multiple flame balls with interesting dynamic behaviour. Some of the observations can be explained with multiple ball solutions of the spherical flame model with the inclusion of heat loss.

The governing equations for a spherical system with heat loss and first order Arrhenius kinetics can be found from the equations for energy conservation and heat and mass balance and can be written as (e.g. Buckmaster et al. [3])

$$
\begin{aligned}
\rho c_{p} \frac{\partial T}{\partial t} & =k \nabla^{2} T+Q A Y e^{-E / R T}-\frac{h S}{V}\left(T-T_{a}\right) \\
\frac{\partial Y}{\partial t} & =D \nabla^{2} Y-A Y e^{-E / R T}
\end{aligned}
$$

These equations are very similar to those written in the previous section, except that they are now in a spherical coordinate system. They are typically 
solved subject to the flame sheet model for the reaction term

$$
A Y e^{-E / R T} \longrightarrow B e^{-E / 2 R T_{*}} \delta\left(r-r_{*}\right),
$$

as in Buckmaster et al. [3], where the constant $B$ is a measure of the strength of the reaction and the internal flame ball temperature is defined to be $T_{*}$. For example, one then finds that the radius of a flame ball, $r_{*}$, is related to the heat loss, $h S / k V$, by

$$
\frac{D Y_{\infty}}{B}=r_{*} \exp \left[-\frac{\left(E / 2 R T_{\infty}\right)}{\left(1+\frac{Y_{\infty} Q}{\operatorname{Le} \rho c_{p} T_{\infty}} \cdot \frac{1}{\left(1+r_{*} \sqrt{h S / k V}\right)}\right)}\right]
$$

The main result from this analysis is the size of the flame ball. One can also use this steady state result to show that radiative or convective heat losses are required to obtain a flame ball solution that is stable; that is the solution with $h=0$ is unstable, while solutions with $h \neq 0$ may be stable. It is also possible to consider scenarios with multiple flame balls. Beginning with two flame balls, one writes down the conservation equations is then interested to determine if it is possible to have two stable, different sized flame balls. The asymptotic analysis becomes rather complicated and the results will be reported in a separate paper, but the answer lies in the solution of a system of two coupled algebraic equations. Solving these for the radii, gives the diagram shown in Figure 3, where the radius of the ball(s) is plotted against the heat loss. The solid curve is the result for the two 
balls being of the same size. The dashed line shows a bifurcation suggesting two different sized flame balls co-existing for a selected range of parameter values, but all the currently available numerical and experimental evidence suggests that this is an unstable situation.

\section{Conclusion}

In this paper we have shown three different instances of bifurcation occurring in mathematical models of combustion phenomena. There are many others that could also be added to this list, from well stirred reactors to flames located in stagnation point flows. Hopf bifurcations and period doubling routes to chaos are among the familiar signatures of chaos that can be found in various forms of these models. In all of them, to understand the dynamical behaviour and its relevance to combustion phenomena, it is necessary to first carry out some analytical work before embarking upon computations.

\section{References}

[1] A. Bayliss and B. J. Matkowsky. From traveling waves to chaos in combustion. SIAM J. Appl. Math., 54(1):147-174, February 1994.

C1471, C1474 


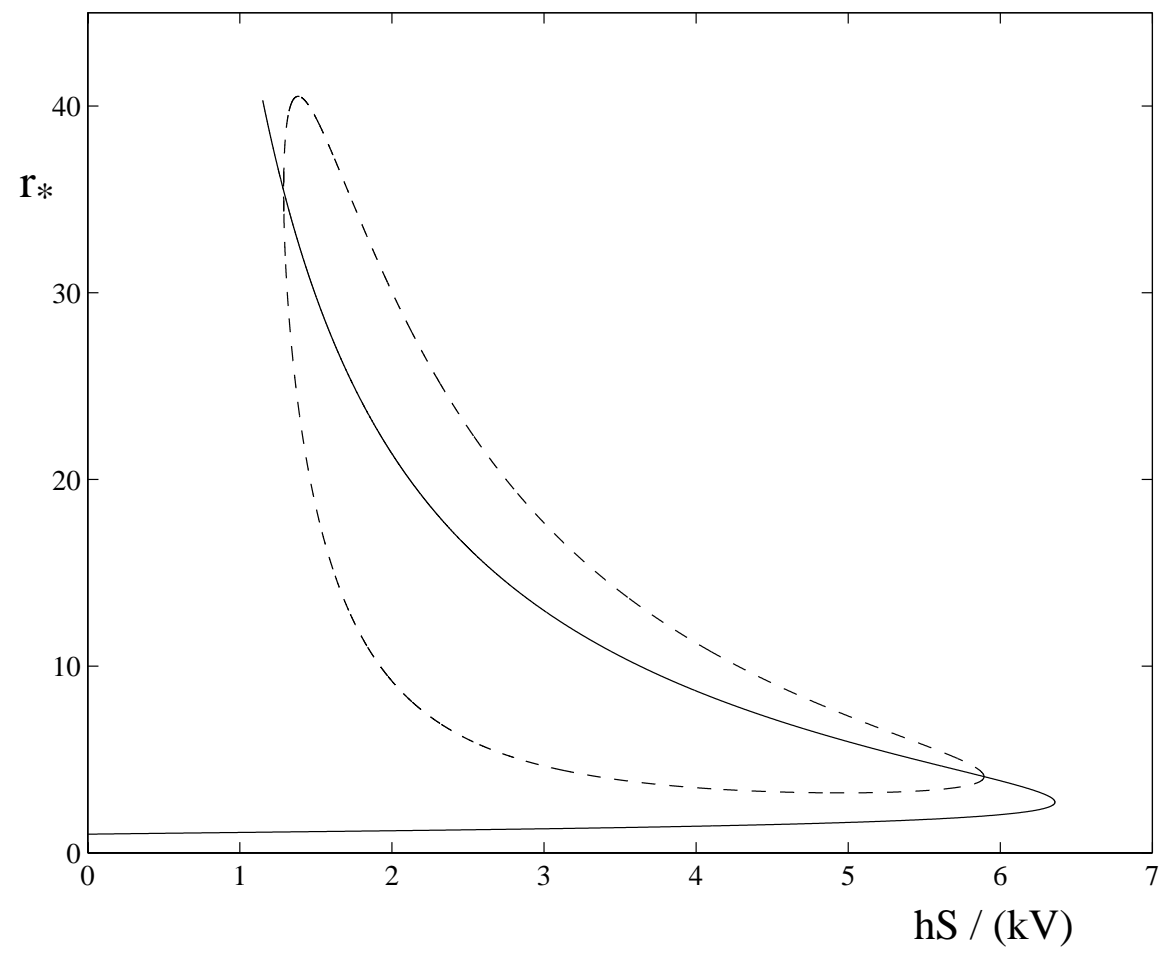

Figure 3: Flame ball size, $r_{*}$, versus heat loss parameter, $h S / k V$. 
[2] J. Buckmaster, G. Joulin, and P. Ronney. The structure and stability of nonadiabatic flame balls. Combustion and Flame, 79:381-392, 1990. C1476

[3] J. Buckmaster, M. Smooke, and V. Giovangigli. Analytical and numerical modeling of flame-balls in hydrogen-air mixtures. Combustion and Flame, 94:113-124, 1993. C1476, C1477

[4] E.J. Doedel, A.R. Champneys, T.F. Fairgrieve, Yu.A. Kuznetsov, B. Sandstede, and X. Wang. AUTO97: Continuation and bifurcation software for ordinary differential equations (with HomCont). Technical Report, Concordia University, USA, 1997. C1469

[5] D. A. Frank-Kamenetskii. Diffusion and Heat Transfer in Chemical Kinetics. Plenum Press, New York, 2nd edition, 1969. C1467, C1469

[6] D. Lozinski, J. Buckmaster, and P. Ronney. Absolute flammability limits and flame-balls. Combustion and Flame, 97:301-316, 1994. C1476

[7] B. J. Matkowsky and G. I. Sivashinsky. Propagation of a pulsating reaction front in solid fuel combustion. SIAM J. Appl. Math., 35(3):465-478, November 1978. C1471, C1474

[8] C.M. Rivers, G.C. Wake, and X.D. Chen. The role of drying in the spontaneous ignition of moist milk powder. Math. Engng. Ind., 6(1):1-14, 1996. C1469 
[9] K. G. Shkadinskii, B. I. Khaikin, and A. G. Merzhanov. Propagation of a pulsating exothermic reaction front in the condensed phase. Combustion, Explosion and Shock Waves, 1:19-28, 1971. translated from Fizika Goreniya i Vzryva, No. 1, pp 19-28, January-March 1971. C1471

[10] R.O. Weber, E. Balakrishnan, and G.C. Wake. Critical initial conditions for spontaneous thermal ignition. J. Chem. Soc., Faraday Trans., 94:3613-3617, 1998. C1468

[11] R.O. Weber, G.N. Mercer, H.S. Sidhu, and B.F. Gray. Combustion waves for gases $(\mathrm{Le}=1)$ and solids $(\mathrm{Le} \rightarrow \infty)$. Proc. R. Soc. Lond. A, 453:1105-1118, 1997. C1471, C1474, C1475

[12] R.O. Weber, G.C. Wake, H.S. Sidhu, G.N. Mercer, B.F. Gray, W. Derrick, and E. Balakrishnan. On the crossing of intermediate unstable steady state solutions for thermal ignition in the sphere. The ANZIAM Journal, tba:to appear, 2000. C1469

[13] Ya.B. Zeldovich, G.I. Barenblatt, V.B. Librovich, and G.M. Makhviladze. The Mathematical Theory of Combustion and Explosions. Consultants Bureau, Plenum, New York, 1985. C1467, C1476 